\title{
Physiological analysis of stoop lifting based on heart rate for the Malaysian population
}

\begin{abstract}
The growing problem of work related back injuries has contributed to the rising cost of health care, human suffering and loss of productivity. In Malaysia, back pain injury awareness is still low, but the number of casualties as a result of back pain is increasing. In this study, the physiological aspects of stoop lifting posture on heart rate for the Malaysian population were studied. Different lifting heights, frequency, twist angle and weight of loads were studied to identify and recommend the appropriate lifting tasks to be used by the workers. An experimental work was conducted involving 36 subjects between 20 and 40 years old with no history of back pain. The subjects performed stoop lifting posture to lift loads of 10, 15, 20 and $23 \mathrm{~kg}$ to a lifting height of $70 \mathrm{~cm}$ and $130 \mathrm{~cm}$, with the frequency of 1 lift, 3 lifts and 6 lifts per minute and twist angle of $0^{\circ}$ and $90^{\circ}$. The results of the study showed that heart rate is linearly related to height of lifting, weights, frequency and angle of twisting. These findings can be used as a guide to design lifting tasks for the Malaysian population.
\end{abstract}

Keyword: Heart rate; Physiology; Lifting task; Stoop lifting 